\title{
Bioremediation of Waste Water Containing Hazardous Cadmium Ion with Ion Imprinted Interpenetrating Polymer Networks
}

\author{
Girija Parameswaran ${ }^{1}$ and Beena Mathew ${ }^{2}$ \\ ${ }^{1}$ Department of Chemistry, S. D. College, Alappuzha, Kerala 690104, India \\ ${ }^{2}$ Schoolof Chemical Sciences, Mahatma Gandhi University, Kottayam, Kerala 686560, India \\ Correspondence should be addressed to Girija Parameswaran; girijakallelil@gmail.com
}

Received 31 December 2013; Accepted 15 February 2014; Published 16 April 2014

Academic Editor: Jesus Simal-Gandara

Copyright (C) 2014 G. Parameswaran and B. Mathew. This is an open access article distributed under the Creative Commons Attribution License, which permits unrestricted use, distribution, and reproduction in any medium, provided the original work is properly cited.

\begin{abstract}
A novel Cd(II) ion imprinted interpenetrating polymer network (Cd(II)IIP) was prepared by free radical polymerization using alginic acid and NNMBA-crosslinked polyacrylamide in presence of initiator potassium persulphate. Cd(II)IIP showed higher capacity and selectivity than the nonimprinted polymer (NIP). The sorption capacities of Cd(II)IIP and NIP for Cd(II) ions were 0.886 and 0.663 meqmole $^{-1}$, respectively. Kinetics studies showed that the sorption process closely agreed with a pseudosecondorder model. The thermodynamic data suggest that the sorption is a spontaneous endothermic process. Equilibrium experiments showed very good fit with the Langmuir isotherm equation for the monolayer sorption process. Cd(II)IIP exhibited good reusability, and the sorption capacity of Cd(II)IIP was stable within the first 4 cycles without obvious decrease. Also Cd(II)IIP showed almost $100 \%$ removal efficiency for $\mathrm{Cd}(\mathrm{II})$ ions in real environmental water samples, indicating that $\mathrm{Cd}$ (II)IIP could have wide application prospects in $\mathrm{Cd}(\mathrm{II})$ ion removal.
\end{abstract}

\section{Introduction}

Water pollution is one of the most serious environmental problems of the present day. Water obtained from different sources is associated with a large number of impurities. Among them heavy metal toxicity is very crucial. Occurrence of toxic metals in lakes, ponds, ditch, and river water affect the lives of local people that depend on these water sources for their daily requirements [1]. Currently, anthropogenic inputs of metals exceed natural input. High levels of $\mathrm{Cd}, \mathrm{Cu}, \mathrm{Pb}$, and Fe can act as ecological toxins in aquatic and terrestrial ecosystem $[2,3]$. Cadmium, as a hazardous pollutant commonly present in the living environment, represents an important risk to human health. Cadmium and its compounds are highly toxic and exposure to this metal is known to cause cancer and targets the body's cardiovascular, renal, gastrointestinal, neurological, reproductive, and respiratory systems. The approach of bioremediation is an innovative tool with greater potential to remove heavy metal ions in water bodies. The method of ion imprinting has become one of the fast-growing technologies that have achieved a lot of attention recently especially in the area of materials science. The IIPs are synthesized on the principle of enzyme phenomenon whereby a monomer is altered by a polymerization that takes place in the presence of a template that will be later removed to create cavities that will recognize only the analyte of interest. Also, the international agency for research on cancer has classified cadmium as a human carcinogen [4]. Zhai et al. have reported selective solid-phase extraction of trace cadmium(II) ions with an ion imprinted polymer (IIP), prepared by a dual ligand monomer [5]. An imprinted organic/inorganic hybrid sorbent for selective separation of cadmium from aqueous solution has been proposed by $\mathrm{Lu}$ and Yan [6]. Cd(II) ion imprinted polymer containing epoxy resin has been synthesized and characterized by Pan and coworkers [7]. The designing and synthesis of cadmium (II) ion imprinted polymer are particularly important because cadmium is among the 13 toxic metal species on the priority 
pollutant list of the Environmental Protection Agency (EPA) [8]. Alginate is one of the most extensively investigated biopolymer for the removal of heavy metal ions from aqueous solutions [9-11]. There have been few reports on the synthesis of ion imprinted polymers for removal of $\mathrm{Cd}$ (II) from water samples [12-15]. Thus, the extraction, preconcentration, and determination of trace cadmium from the natural water are very important and need much more attention.

In the present study, we aim to fabricate a novel type of $\mathrm{Cd}(\mathrm{II})$ ion imprinted interpenetrating polymer network using a natural biosorbent, alginic acid which is crosslinked by the hydrophilic crosslinker $\mathrm{N}, \mathrm{N}^{\prime}$ methylenebis-acrylamide and template $\mathrm{Cd}(\mathrm{II})$ ion. The hydrophilic nature of crosslinker increased the swelling ability of polymer network which favours effective cadmium ion binding. The newly prepared interpenetrating polymer network is advantageous because it showed high sorption capacity and remarkable selectivity in $\mathrm{Cd}$ (II) ion separation from mixture of metal ions. Compared to the previous reports, the present IIP showed high sorption capacity and good reusability for $\mathrm{Cd}$ (II) ion and the synthesis of IIP involves clean technology and can be used for waste water treatment. The biopolymers are capable of removing metal ions. The method of preparation of the IPN can be considered as "green" and also simple, rapid, low cost, and environment friendly due to the use of aqueous medium.

\section{Materials and Methods}

Reagents of analytical and spectral grade were used for all experiments. Cadmium acetate used in the present study is of analytical grade (99\% pure) and solutions were prepared by dissolving the required quantity of $\mathrm{CdCH}_{3}(\mathrm{COO})_{2}$ in millipore water. The monomers used in this study, namely, acrylamide (99.9\% pure) and crosslinking agent NNMBA, were obtained from SRL (Mumbai). Alginic acid $(98.5 \%$ pure) was obtained from Merk (India). Fourier transform infrared (FT-IR) spectra of the imprinted, nonimprinted, and the $\mathrm{Cd}(\mathrm{II})$ ion bound polymer networks were recorded between 4000 and $400 \mathrm{~cm}^{-1}$, using a Perkin Elmer 400 FTIR spectrophotometer and far IR spectra of Cd(II) ion bound polymer networks were recorded between 400 and $100 \mathrm{~cm}^{-1}$ using the same instrument. UV-vis spectrophotometric measurements were carried out using Shimadzu 2400 UV-vis spectrophotometer. SEM-EDAX were taken on JEOL-JSM$840 \mathrm{~A}$ scanning electron microscope in nitrogen atmosphere. TG curves were recorded on a Shimadzu D-740 thermal analyser at a heating rate of $10^{\circ} \mathrm{C}$ min from 20 to $900^{\circ} \mathrm{C}$ in nitrogen atmosphere. The amount of metal ion sorbed per gram of imprinted and nonimprinted polymers from metal ion solutions was determined, using Perkin Elmer atomic absorption analyzer 300 .

2.1. Preparation of Cd(II) Ion Imprinted (IIP) and Nonimprinted (NIP) Polymer Networks. Alginic acid (7.5 g) was mixed with $(0.64 \mathrm{~g})$ of cadmium acetate in aqueous medium. This mixture was added to an aqueous solution of acrylamide (10.66 g) and N,N-methylenebisacrylamide
(NNMBA) (7.71g) and kept at $70^{\circ} \mathrm{C}$ in a water bath with stirring, using potassium persulphate $(100 \mathrm{mg})$ as initiator. The polymer obtained was washed with water to remove unreacted monomer and with $2 \mathrm{~N} \mathrm{HCl}$ to remove $\mathrm{Cd}$ (II) ion. The bulk polymer obtained was dried, sieved, and weighed. Nonimprinted polymer networks were also prepared using the same procedure without metal ions.

2.2. Metal Ion Binding. In order to investigate specific rebinding capacity, $\mathrm{Cd}(\mathrm{II})$ ion imprinted and nonimprinted polymers (500 mg) were equilibrated with $\mathrm{Cd}(\mathrm{II}), \mathrm{Co}(\mathrm{II}), \mathrm{Cu}(\mathrm{II})$, and $\mathrm{Ni}$ (II) (1-5 ppm, $10 \mathrm{~mL})$ ion solution. The concentration of template metal ion solution before and after binding was determined by atomic absorption spectrophotometry (AAS).

2.3. Swelling Studies. $100 \mathrm{mg}$ of Cd(II) ion imprinted, nonimprinted, and corresponding $\mathrm{Cd}$ (II) bound polymers was allowed to swell in $10 \mathrm{~mL}$ water for $24 \mathrm{~h}$. After $24 \mathrm{~h}$, the polymers were filtered and surface water was carefully wiped off, and the final swollen weight was determined. From the swollen and the dry weights of the sample, the EWC (\%) was calculated, using the following equation:

$$
\begin{aligned}
& \operatorname{EWC}(\%) \\
& =\frac{\text { weight of wet polymer }- \text { weight of dry polymer }}{\text { weight of dry polymer }} \times 100 \text {. }
\end{aligned}
$$

2.4. Optimization of the Cd(II) Ion Rebinding Conditions. In order to optimize the conditions of $\mathrm{Cd}(\mathrm{II})$ ion rebinding by imprinted and nonimprinted polymers, factors affecting rebinding such as effect of concentration, time, and $\mathrm{pH}$ of the $\mathrm{Cd}(\mathrm{II})$ ion solution on binding were investigated.

2.5. Effect of Concentration. The batch-wise metal ion binding experiments were carried out using $(500 \mathrm{mg})$ of imprinted and nonimprinted polymer to evaluate the effect of concentration of template solution on rebinding.

From the difference in concentration of template solution before and after incubation, the amount of $\mathrm{Cd}(\mathrm{II})$ ion bound was determined. Similar rebinding studies at various concentrations of metal ion $(1-5 \mathrm{ppm}, 10 \mathrm{~mL})$ were carried out and analyzed by AAS.

2.6. Effect of Time. The time required for maximum binding was determined by batch equilibration method. $500 \mathrm{mg}$ of imprinted and nonimprinted polymers was equilibrated with (5 ppm, $10 \mathrm{~mL}$ ) of $\mathrm{Cd}(\mathrm{II})$ ion solution and metal ion bound was determined at regular intervals of time and analyzed by AAS.

2.7. Effect of $p H$ on Cd(II) Ion Binding. About $100 \mathrm{mg}$ of imprinted and nonimprinted polymers was equilibrated with $\mathrm{Cd}(\mathrm{II})$ ion $(5 \mathrm{ppm}, 10 \mathrm{~mL})$ at different $\mathrm{pH}$. After the removal of polymer particles, the amounts of Cd(II) ion bound at each $\mathrm{pH}$ were determined by AAS. 
2.8. Sorption Studies. Aqueous solution of Cd(II) ion (5 ppm, $10 \mathrm{~mL}$ ) was added to $(100 \mathrm{mg})$ of imprinted and nonimprinted polymers. The solutions were shaken in stoppered bottles. At regular time intervals, the concentration of $\mathrm{Cd}(\mathrm{II})$ ion was found out by atomic absorption spectrophotometry. Sorption capacity was investigated using Langmuir's and Freundlich's isotherms.

2.9. Sorption Kinetics Models. Different sets of imprinted polymer $(100 \mathrm{mg})$ were equilibrated with $\mathrm{Cd}(\mathrm{II})$ ion solution $(5 \mathrm{ppm}, 10 \mathrm{~mL})$ at room temperature using a thermostat. After removing the polymer particles, the remaining concentration of $\mathrm{Cd}(\mathrm{II})$ ion was determined at equal intervals of time and analyzed by AAS. To describe the adsorption kinetic behavior of $\mathrm{Cd}(\mathrm{II})$ ion imprinted polymer network, two types of kinetic models were tested, namely, the pseudofirst-order model and pseudosecond-order model. The binding agrees with pseudosecond-order equation.

2.10. Effect of Temperature. The effect of temperature on the sorption of $\mathrm{Cd}(\mathrm{II})$ ion on imprinted polymer network was investigated. Imprinted polymer and nonimprinted polymer $(100 \mathrm{mg})$ were equilibrated with cadmium ion solution $(10 \mathrm{~mL}, 5 \mathrm{ppm})$, at temperature varying from 25 to $40^{\circ} \mathrm{C}$. The $\mathrm{Cd}(\mathrm{II})$ ion bound at each temperature was determined by AAS. The thermodynamic parameters such as $\Delta G^{o}, \Delta H^{o}$, and $\Delta S^{\circ}$ were calculated by applying the van't Hoff equation. Consider

$$
\ln K_{d}=-\frac{\Delta H^{o}}{R T}+\frac{\Delta S^{o}}{R}
$$

where $\Delta G^{o}$ is change in free energy ( $\left.\mathrm{kJmole}{ }^{-1}\right), \Delta H^{o}$ is the change in enthalpy $\left(\mathrm{kJmole}^{-1}\right), \Delta S^{o}$ is the change in entropy $\left(\mathrm{kJmole}{ }^{-1} \mathrm{~K}^{-1}\right), T$ is the absolute temperature $(\mathrm{K}), R$ is the gas constant, and $K_{d}$ is the equilibrium constant.

2.11. Selectivity Studies. Selectivity studies were carried out by column experiment. Cd(II) ion imprinted polymer (1g) was slurred with demineralized water (DMW) and then poured into a Pyrex glass column (id. $40 \mathrm{~mm}$ ) plucked with small portion of glass wool at the bottom. The column was preconditioned by passing DMW followed by the mixture of metal ion solution $(5 \mathrm{ppm}, 10 \mathrm{~mL})$ that was passed through the column at a flow rate of $\sim 0.5 \mathrm{~mL} \mathrm{~min}^{-1}$. The eluted solution was collected and the amount of metal ion bound was determined by atomic absorption spectrophotometric method.

\section{Results and Discussion}

3.1. Synthesis of Cd(II) Ion Imprinted and Nonimprinted Interpenetrating Polymer Networks. The Cd(II) ion imprinted polymer networks were synthesized by free radical polymerization of acrylamide and N,N-methylenebisacrylamide (NNMBA) in presence of alginic acid. Potassium persulphate $(100 \mathrm{mg})$ was used as initiator and $\mathrm{Cd}(\mathrm{II})$ ion was used as template, and the polymerization was carried out at $70^{\circ} \mathrm{C}$

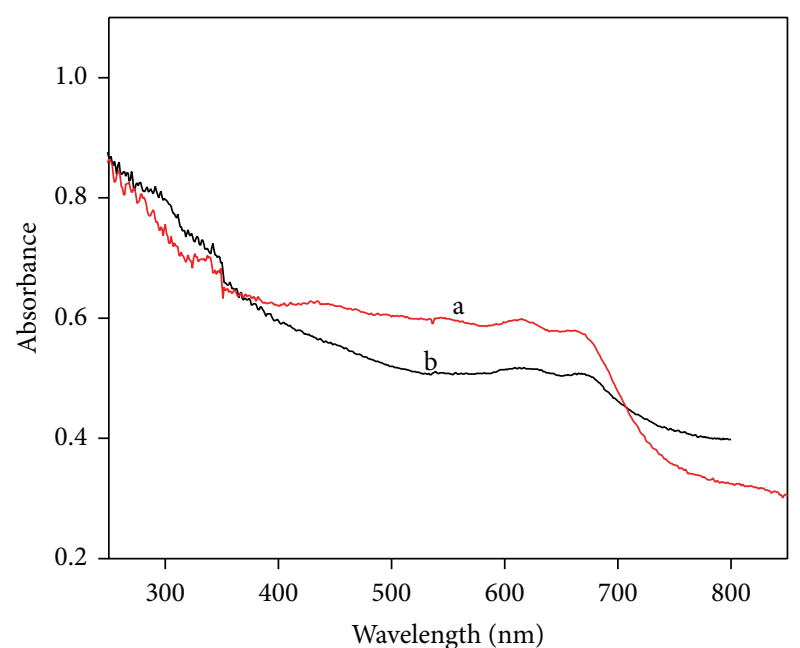

FIGURE 1: UV-vis spectra of (a) Cd(II) ion desorbed and (b) Cd(II) ion bound imprinted polymers.

(Scheme 1). The bulk polymer obtained was washed with water to remove unreacted monomers and with dilute $\mathrm{HCl}$ to remove $\mathrm{Cd}(\mathrm{II})$ ions. The polymer was dried, crushed, and sieved. Nonimprinted polymer networks were also prepared without using the template metal ion.

\subsection{Characterization of NNMBA-Crosslinked Cd(II) Ion Imprinted and Nonimprinted Interpenetrating Polymer Networks}

3.2.1. FT-IR Spectra. FT-IR spectrum of the IPN is different from those of the pure polymers because in interpenetrating polymer networks there will be intermolecular interactions. The carboxylate group plays an important role in metal sorption property of alginic acid. FT-IR spectra of $\mathrm{Cd}(\mathrm{II})$ ion imprinted polymer networks showed absorption bands at $1643 \mathrm{~cm}^{-1}$ which is assigned to carboxylate group of alginic acid. This band is shifted to $1634 \mathrm{~cm}^{-1}$ after Cd(II) ion binding. This result showed that $\mathrm{Cd}(\mathrm{II})$ ion binding takes place at carboxylate group of alginate. Imprinted polymer showed bands at $2923 \mathrm{~cm}^{-1}$ and nonimprinted polymer showed bands at $2953 \mathrm{~cm}^{-1}$ due to $\mathrm{C}-\mathrm{H}$ stretching vibrations.

3.2.2. UV-Vis Spectra. UV-vis spectra of Cd(II) ion imprinted interpenetrating polymer networks and the corresponding $\mathrm{Cd}(\mathrm{II})$ ion bound polymer networks are shown in Figure 1. $\mathrm{Cd}(\mathrm{II})$ ion imprinted polymer showed bands at 615,544 , and $434 \mathrm{~nm}$. After Cd(II) ion binding imprinted polymer, these bands are shifted to 663,623 , and $540 \mathrm{~nm}$. Similar trends are seen in nonimprinted polymers. The shift in wavelength to higher region indicates structural changes that occurred on molecules. As a result of interaction of $\mathrm{Cd}(\mathrm{II})$ ion with nonbonding electrons in the carboxylate group of alginic acid, a shift in the wavelength maxima takes place. This might be due to $n \rightarrow \pi^{*}$ transitions. Ligand to metal charge transfer bands are obtained at 294 and $327 \mathrm{~nm}$ in imprinted and $\mathrm{Cd}(\mathrm{II})$ ion bound polymers. 


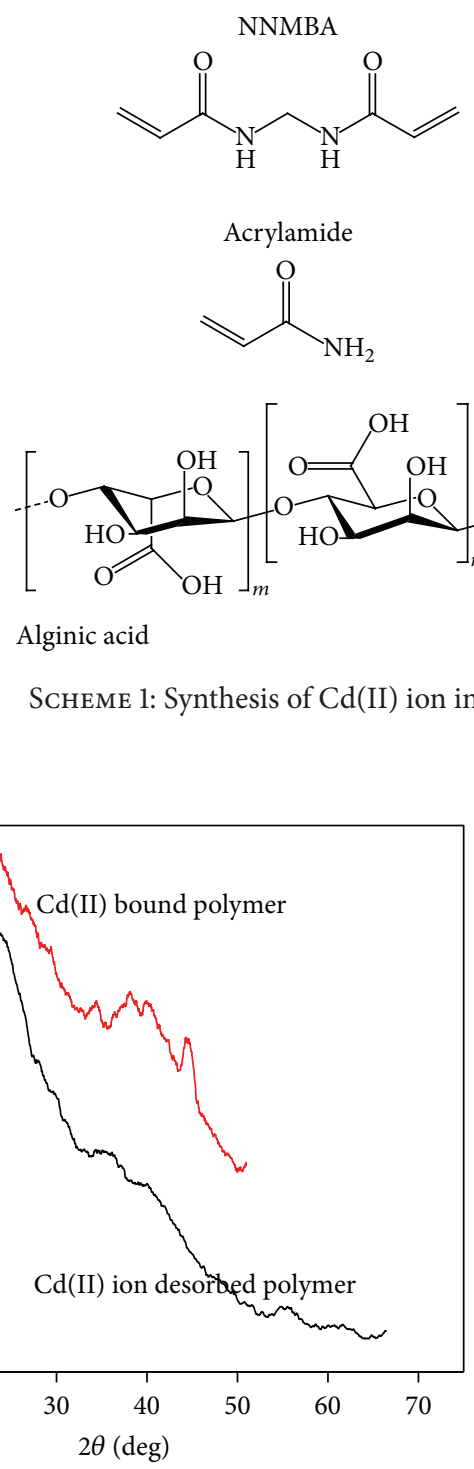

FIGURE 2: XRD pattern of imprinted and Cd(II) bound polymers.

3.3. X-Ray Diffraction Patterns. X-ray diffraction patterns of $\mathrm{Cd}(\mathrm{II})$ ion imprinted and bound polymers are shown in Figure 2. The XRD patterns of both polymers exhibited similar patterns, which indicate an amorphous nature. Generally, the amorphous compounds are denoted by very broad halo peaks. XRD patterns of both imprinted and metal ion bound polymers did not show any strong reflection peaks in the 2 theta regions 20 to $60^{\circ}$, indicating the amorphous nature for $\mathrm{Cd}(\mathrm{II})$ ion bound and unbound polymers.

3.4. SEM-EDAX. The chemical composition of the imprinted and nonimprinted polymer networks were confirmed by SEM-EDAX. The presence and complete removal of Cd(II) ion in imprinted and $\mathrm{Cd}(\mathrm{II})$ bound polymers, respectively, were confirmed by SEM-EDAX. As shown in Figure 3, the signal due to $\mathrm{Cd}(\mathrm{I1})$ was clearly presented for $\mathrm{Cd}$ (II) bound imprinted polymer but was absent in cadmium ion desorbed polymer.
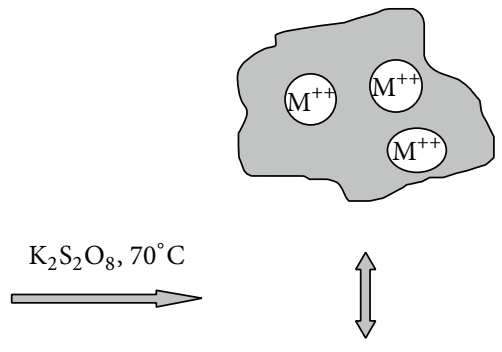

IIP

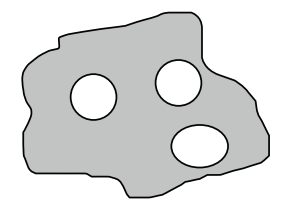

ing polymer networks.

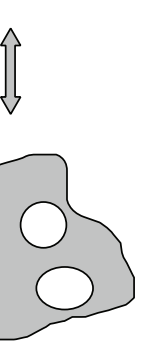

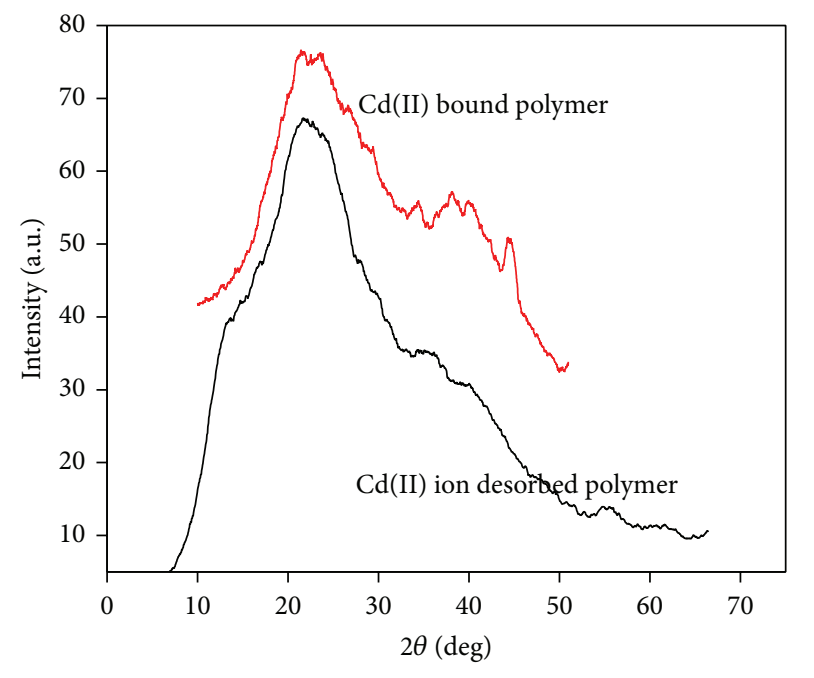




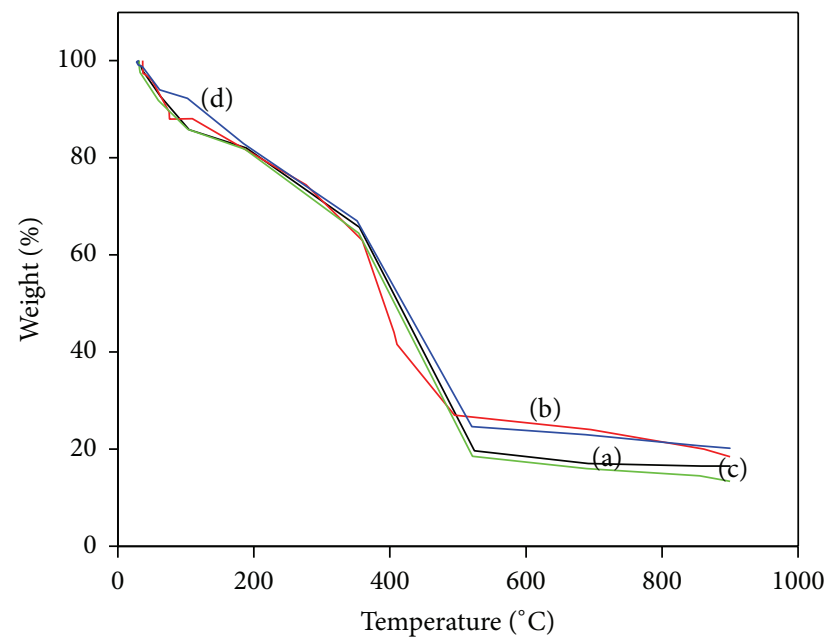

$\begin{array}{ll}\text { (a) Imprinted polymer } & \text { (c) } \mathrm{Cd} \text { (II) bound nonimprinted polymer }\end{array}$ (b) Cd(II) bound polymer (d) Nonimprinted polymer

FIGURE 4: TGA curves of Cd(II) ion imprinted, nonimprinted, and Cd(II) ion bound IPNs.

uncomplexed ligands. The second stage is the major decomposition in which polymer chain breaks leaving only the metallic residue. Thermogravimetric analysis of imprinted polymer showed $15 \%$ weight loss at about $80-120^{\circ} \mathrm{C}$, which is ascribed to the removal of carboxylate group or uncomplexed ligands. During the second stage of decomposition at 300$400^{\circ} \mathrm{C}, 50 \%$ weight loss is observed which is attributed to the decomposition of polymer chain, while in $\mathrm{Cd}(\mathrm{II})$ bound polymer the decomposition was in the range $150-500^{\circ} \mathrm{C}$, resulting in a mass loss of $55 \%$. Nonimprinted polymer networks also decompose in a similar manner. The temperatures for maximum weight loss $\left(T_{\max }\right)$ for imprinted polymer and Cd (II) ion bound polymer networks were 310 and $390^{\circ} \mathrm{C}$, respectively. From the Figure 4, it is clear that thermal stability of Cd(II) bound interpenetrating polymer networks is much higher than that of uncomplexed polymers.

3.6. Swelling Studies. The efficiency of a functional polymer is governed by the accessibility of the reactive functional groups anchored on it, which in turn depends on the extent of swelling and solvation [17]. The metal ion binding studies of the interpenetrating polymer networks in aqueous medium were influenced by the extent of swelling. A good solvent brings the crosslinked polymer to a state of complete solvation and the polymer network can expand to form a gel. The extent of swelling was dependent on the nature of the polymer backbone, molecular character, and extent of crosslinking agent. The swelling behavior of $\mathrm{Cd}(\mathrm{II})$ ion imprinted and nonimprinted polymers and their corresponding $\mathrm{Cd}(\mathrm{II})$ bound polymer networks was investigated (Table 1). Maximum EWC (\%) was obtained for Cd(II) ion imprinted polymer and it decreases on metal complexation.

3.7. Binding Studies with Metal Ions. The effect of initial concentration of metal ion solution on its sorption was investigated by varying the concentration of the metal ions, such
TABLE 1: EWC (\%) values of imprinted and nonimprinted polymers and their $\mathrm{Cd}(\mathrm{II})$ ion bound polymers.

\begin{tabular}{lc}
\hline Polymers used & EWC (\%) \\
\hline IIP & 88 \\
NIP & 86 \\
Cd(II) bound IIP & 85 \\
Cd(II) bound NIP & 84 \\
\hline
\end{tabular}

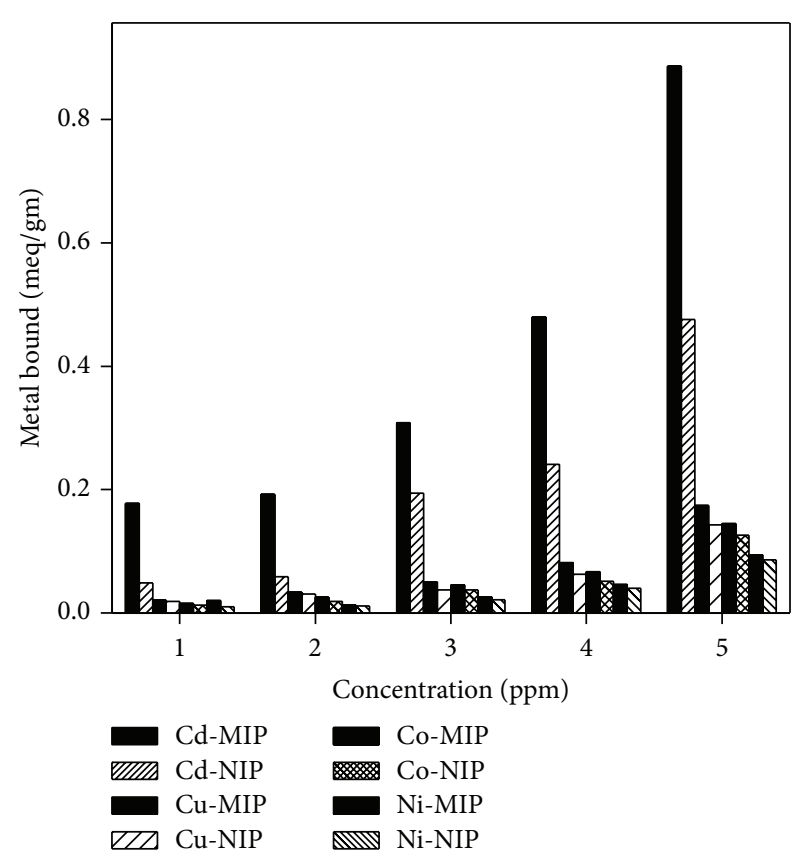

FIGURE 5: Effect of concentration of metal ion solution on its binding by imprinted and nonimprinted polymers.

as $\mathrm{Cd}(\mathrm{II}), \mathrm{Co}(\mathrm{II}), \mathrm{Cu}(\mathrm{II})$, and $\mathrm{Ni}(\mathrm{II})$ ions. The imprinted and nonimprinted polymer networks $(500 \mathrm{mg}$ ) were equilibrated with metal ion solution $(1-5 \mathrm{ppm}, 10 \mathrm{~mL})$; the concentration of template before and after binding was determined by atomic absorption spectrophotometry. It was noted that, as the concentration increases, binding of metal ion increases (Figure 5). This result could be explained on the basis of a high driving force for mass transfer, where the increase in concentration of metal ion increases the competition to occupy all the available coordination sites in the adsorbent.

3.8. Effect of Time on Metal Ion Binding. To optimize the time taken for maximum binding of Cd(II) ion by imprinted and nonimprinted polymer networks, $100 \mathrm{mg}$ of polymer networks was equilibrated with $\mathrm{Cd}(\mathrm{II})$ ion solution $(10 \mathrm{~mL}$, $5 \mathrm{ppm}$ ) and the binding was followed by AAS at definite intervals of time. The time dependence of adsorption capacities of $\mathrm{Cd}(\mathrm{II})$ ions on imprinted and nonimprinted polymers was given in Figure 6. High adsorption rates were observed at the beginning of the adsorption process, after which adsorption equilibrium was quickly reached within $90 \mathrm{~min}$ for imprinted polymer network and $80 \mathrm{~min}$ for nonimprinted polymer network. The imprinted polymer networks possessing complementary binding site required more time to 


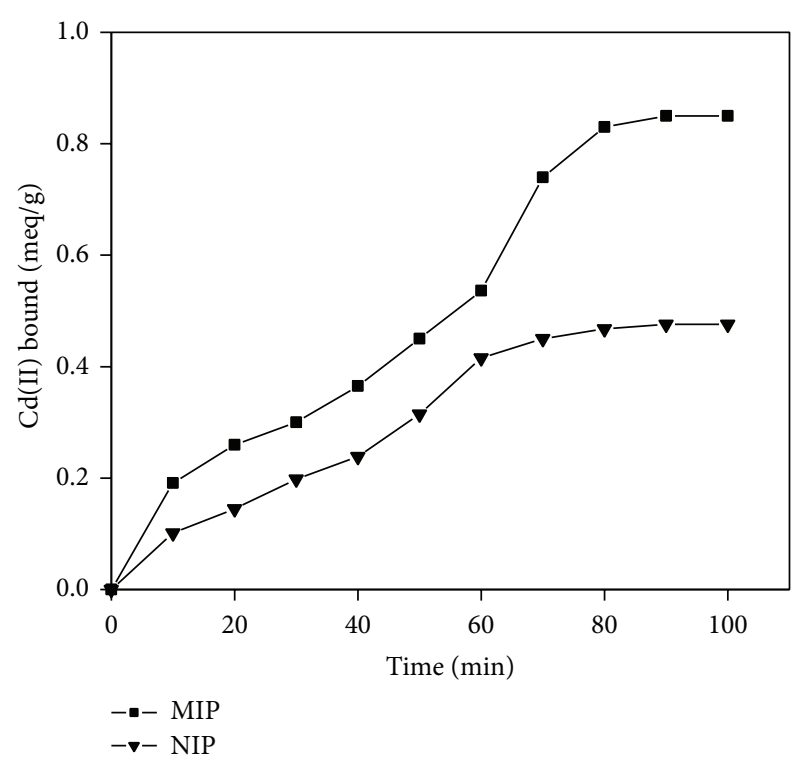

FIGURE 6: Effect of time on Cd(II) ion binding by imprinted and nonimprinted polymers.

attain saturation, since the template metal ion has to penetrate through highly crosslinked interpenetrating polymer networks. In nonimprinted polymer networks, there is no such specific arrangement of the binding sites. The maximum adsorption capacity for $\mathrm{Cd}(\mathrm{II})$ ions was $0.87 \mathrm{meqg}^{-1}$ of imprinted polymers. This fast adsorption equilibrium is due to high complexation rate and matching of the incoming $\mathrm{Cd}(\mathrm{II})$ ions and $\mathrm{Cd}(\mathrm{II})$ cavities in the network structure.

3.9. Effect of $\mathrm{pH}$ on Metal Ion Binding. The $\mathrm{pH}$ of the medium is one of the most important factors controlling the sorption of metal ions by adsorbent. The sorption of Cd(II) ion uptake at different $\mathrm{pH}$ was examined at equilibration by equilibrating imprinted and nonimprinted polymer $(100 \mathrm{mg})$ with $(10 \mathrm{~mL}, 5 \mathrm{ppm})$ metal ion solution at varying $\mathrm{pH}$ and the metal ion bound was determined by AAS. Sorption of metal ion increases with increase in $\mathrm{pH}$ of the medium and then decreases (Figure 7). It could be attributed to the fact that, at low $\mathrm{pH}$, metal competition between the metal ion and the $\mathrm{H}^{+}$ion favors protonation of carboxylate groups, thus lowering the metal ion binding. But at higher $\mathrm{pH}$ of the medium, the protonation decreases and as a result metal ion uptake increases.

3.10. Sorption Studies. The effect of concentration of Cd(II) ion solution on sorption rate and capacity was studied. Definite amounts of imprinted and nonimprinted polymers were added to fixed amount of Cd(II) ion solution (5 ppm). The solutions were shaken in closed flasks. At regular intervals of time, Cd(II) ion bound was determined by AAS. The sorption characteristics were assessed by plotting both Langmuir's and Freundlich's isotherms. The Langmuir equation can be written as

$$
\frac{C_{e}}{q_{e}}=\frac{C_{e}}{q_{o}}+\frac{1}{b q_{o}}
$$

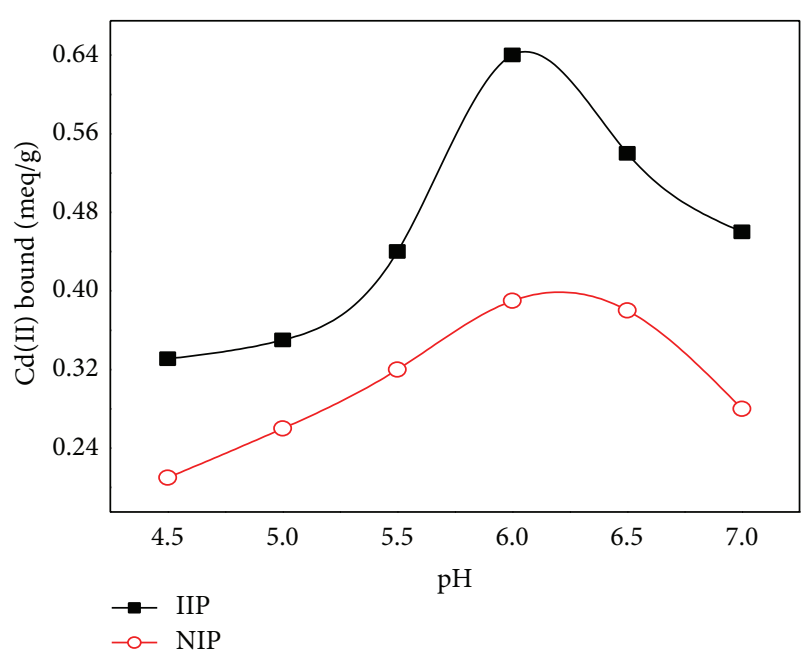

FIgURE 7: Effect of $\mathrm{pH}$ on $\mathrm{Cd}(\mathrm{II})$ ion binding.

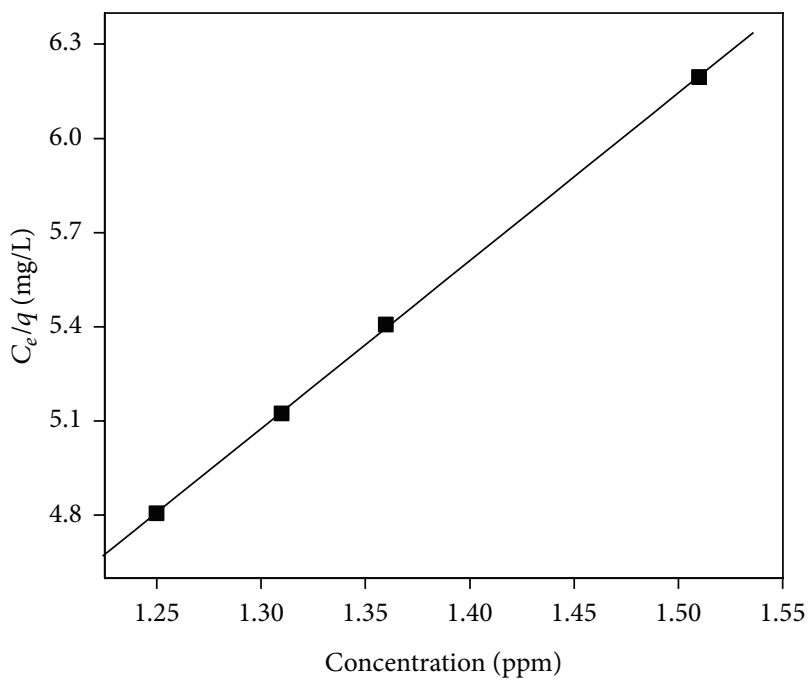

FIGURE 8: Langmuir isotherm of Cd(II) ion imprinted polymer network.

where $C_{e}\left(\mathrm{mgL}^{-1}\right)$ is the equilibrium concentration, $q_{e}$ $\left(\mathrm{meqg}^{-1}\right)$ is the adsorption amount in equilibrium, $q_{o}$ is the maximum adsorption amount, and $b$ is the Langmuir constant related to adsorption capacity and energy of adsorption.

For Langmuir's isotherm, $C_{e} / q_{e}$ is plotted against $C_{e}$, and a straight line graph with $R^{2}$ value 0.9984 was obtained (Figure 8).

Freundlich's equation can be written as

$$
\log q_{e}=\log K+(1-n) \log C_{e},
$$

where $K$ is the Freundlich's constant and $n$ is the Freundlich's exponent.

For Freundlich's isotherm, $\log q_{e}$ is plotted against $\log C_{e}$, and a straight line graph with correlation coefficient 0.944 was obtained (Figure 9). By comparison of the adsorption isotherms of cadmium ion imprinted polymer with Langmuir's and Freundlich's models, it is found that 


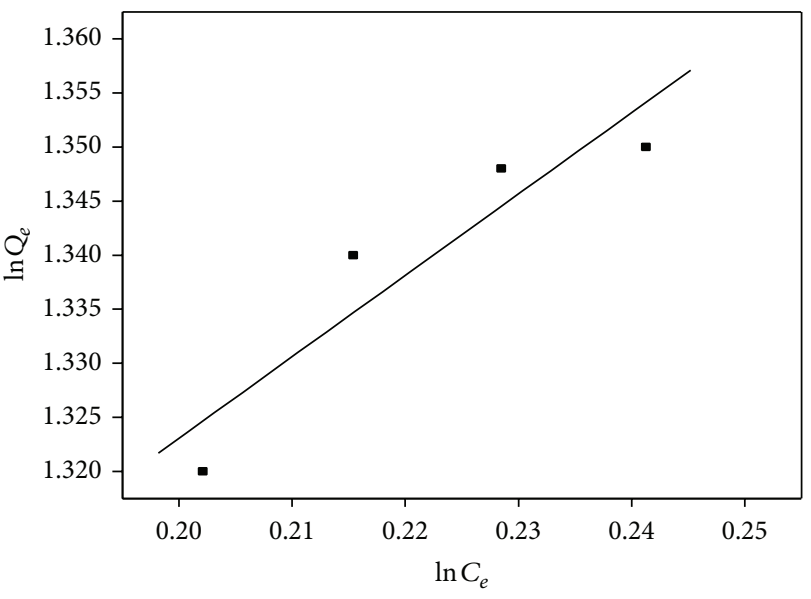

Figure 9: Freundlich's isotherm.

the experimental data is in agreement with Langmuir's isotherm better than that of Freundlich's isotherm.

\subsection{Langmuir Isotherm. See Figures 8 and 9.}

3.12. Effect of Temperature. The effect of temperature on the adsorption process was investigated for the adsorption of cadmium ion on the imprinted polymer by batch equilibration method and the amount of template bound at each temperature was determined by AAS. The temperature was varied from 25 to $40^{\circ} \mathrm{C}$. The obtained results showed that increase in temperature favours the sorption process. Thermodynamic parameters were calculated using the following equation:

$$
\ln K_{d}=-\frac{\Delta H^{o}}{R T}+\frac{\Delta S^{o}}{R}
$$

where

$$
\begin{gathered}
K_{d}=\frac{Q_{e}}{C_{e}}, \\
\Delta G^{o}=\Delta H^{o}-T \Delta S^{o}, \\
\Delta G^{o}=-R T \ln K_{d},
\end{gathered}
$$

where $\Delta G^{o}$ is change in free energy $\left(\mathrm{kJmole}{ }^{-1}\right), \Delta H^{o}$ is the change in enthalpy $\left(\mathrm{kJmole}^{-1}\right), \Delta S^{o}$ is the change in entropy $\left(\mathrm{kJmole}^{-1} \mathrm{~K}^{-1}\right.$ ), $T$ is the absolute temperature $(\mathrm{K}), R$ is the gas constant, and $K_{d}$ is the equilibrium constant.

When $\log K_{d}$ is plotted against $I / T$, a straight line graph is obtained with slope $-\Delta H^{o} / 2.303 R T$ and the values of $\Delta H^{o}$ and $\Delta S^{\circ}$ were obtained from the slope and intercept of the van't Hoff plot (Figure 10). The values of $\Delta H^{o}, \Delta G^{o}$, and $\Delta S^{o}$ for the plot shown in Figure 10 are found to be 165.2, -204 $\mathrm{kJmole}^{-1}$, and $232.6 \mathrm{kJmole}{ }^{-1} \mathrm{~K}^{-1}$. The negative value of $\Delta G^{o}$ indicates the feasibility and spontaneous nature of sorption process. The value of $\Delta H^{o}$ was positive indicating that the binding was endothermic in nature and the positive value of $\Delta S^{o}$ suggested an increase in randomness during the sorption process.

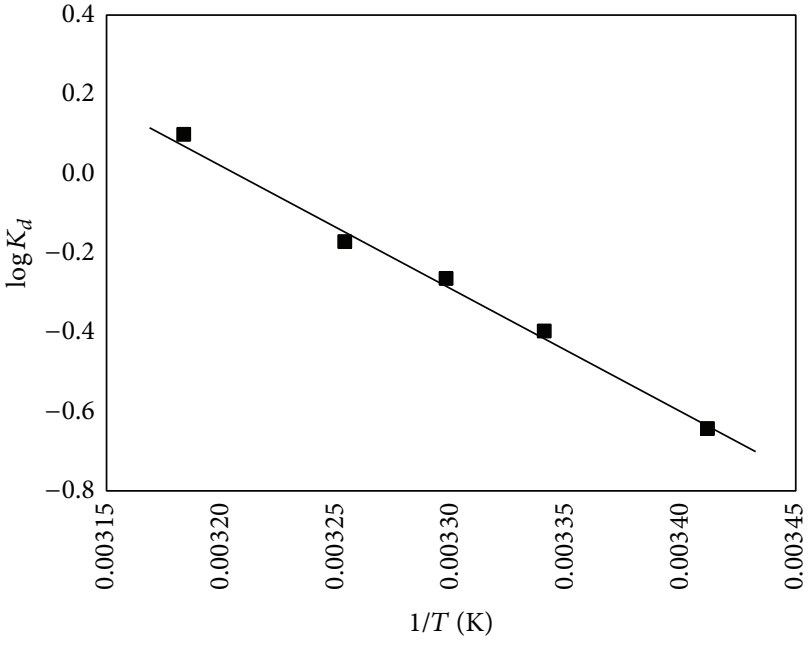

Figure 10: The linear van't Hoff equation.

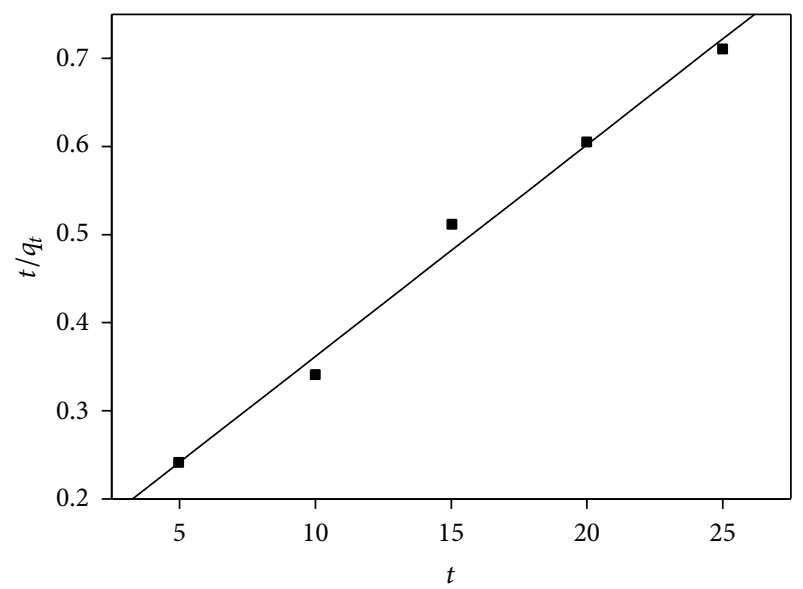

FigURE 11: Kinetic model of pseudosecond order.

3.13. Sorption Kinetics. The pseudosecond-order Lagergren equation was used to describe the sorption kinetics of cadmium ion imprinted IPN as described earlier. The sorption kinetics of cadmium ion imprinted IPN confirmed to the pseudosecond-order Lagergren equation with $R^{2}=0.999$ (Figure 11). The value of $K_{2}$ calculated from the slope is $0.0412 \mathrm{~min}^{-1}$.

The pseudosecond-order Lagergren equation was used to describe the adsorption kinetics of cadmium ion imprinted IPN. Consider

$$
\frac{t}{q_{t}}=\left(\frac{1}{K_{2} q_{e}^{2}}+\frac{t}{q_{e}}\right),
$$

where $K_{2}\left(\right.$ gmgmin $\left.^{-1}\right)$ is the second-order Lagergren constant of adsorption and $q_{t}\left(\mathrm{meqg}^{-1}\right)$ and $q_{e}\left(\mathrm{meqg}^{-1}\right)$ are the quantities of metal ions adsorbed at time $t(\mathrm{~min})$ and at equilibrium, respectively. The adsorption kinetics of cadmium ion imprinted IPN was confirmed to the pseudosecondorder Lagergren equation with $R^{2}=0.999$. The value of $K_{2}$ calculated from the slope is $0.0412 \mathrm{~min}^{-1}$. 
TABLE 2: Selectivity parameters of Cd(II) ion imprinted polymers.

\begin{tabular}{lccc}
\hline Metal ions & $\alpha_{i}$ & $\alpha_{n}$ & $\alpha_{r}$ \\
\hline $\mathrm{Cd}(\mathrm{II}), \mathrm{Zn}(\mathrm{II})$ & 1.90 & 0.30 & 6.30 \\
$\mathrm{Cd}(\mathrm{II}), \mathrm{Ni}(\mathrm{II})$ & 1.64 & 1.01 & 1.62 \\
$\mathrm{Cd}(\mathrm{II}), \mathrm{Mn}(\mathrm{II})$ & 1.06 & 0.71 & 1.51 \\
$\mathrm{Cd}(\mathrm{II}), \mathrm{Cu}(\mathrm{II})$ & 1.01 & 0.92 & 1.11 \\
$\mathrm{Cd}(\mathrm{II}), \mathrm{Co}(\mathrm{II})$ & 1.01 & 0.85 & 1.18 \\
\hline
\end{tabular}

3.14. Selectivity Experiments. The selectivity of the imprinted polymer for $\mathrm{Cd}(\mathrm{II})$ ion was investigated by rebinding $\mathrm{Cd}(\mathrm{II})$ ion in presence of various competitor metal ions (Figure 12). Some important parameters including adsorption capacity, distribution ratio, selectivity factor of Cd(II) ion with respect to the other ions, and relative selectivity factor were calculated (Table 2) from the following equations:

$$
\begin{gathered}
\alpha=\frac{D_{\mathrm{Cd}}}{D_{M}}, \\
D=\frac{Q}{C_{e}}, \\
\alpha_{r}=\frac{\alpha_{i}}{\alpha_{n}},
\end{gathered}
$$

where $Q$ represents the sorption capacity $\left(\mathrm{meqg}^{-1}\right)$ and $C_{e}$ is the equilibrium concentration of metal ions $\left(\mathrm{mg} \mathrm{L}^{-1}\right)$. $D_{\mathrm{Cd}}$ and $D_{M}\left(\mathrm{mLg}^{-1}\right)$ represent the distribution ratios of $\mathrm{Cd}(\mathrm{II})$ and other competitive ions, respectively; $\alpha_{r}, \alpha_{i}$, and $\alpha_{n}$ represent the relative selectivity coefficient, the selectivity factor of imprinted sorbent, and the selectivity factor of nonimprinted sorbent, respectively.

To investigate the $\mathrm{Cd}(\mathrm{II})$ ion selectivity of the imprinted polymer networks, competitive sorption of $\mathrm{Mn}$ (II), $\mathrm{Co}$ (II), $\mathrm{Ni}(\mathrm{II}), \mathrm{Cu}(\mathrm{II})$, and $\mathrm{Zn}(\mathrm{II})$ ions was carried out by column experiment, in which $1 \mathrm{~g}$ of imprinted polymer was treated with $(10 \mathrm{~mL}, 5 \mathrm{ppm})$ solution of these metal ions. After sorption equilibrium was reached, the concentration of metal ions in the remaining solution was measured by AAS. The functional host molecules on the imprinted polymer networks are immobilized with the strict configuration suitable for cadmium ions, and the ionic recognition is influenced by the nature of metal ion and ionic radius and charge. The results revealed that $\mathrm{Cd}(\mathrm{II})$ ion imprinted polymer networks showed high selectivity towards cadmium ion from cadmium-zinc mixture, and maximum separation is obtained depending on the selectivity coefficient. In cadmium-copper mixture, low selectivity coefficient value is obtained. But from the cadmium-nickel mixture, the ion imprinted polymer showed considerable selectivity (Figure 12).

3.15. Reusability Studies. Reusability of cadmium ion imprinted polymers was investigated by elution operations and the results are given in Figure 13. The elution operations were carried out with $4 \mathrm{~mL}$ of $\mathrm{HCl}(3 \mathrm{~N})$ and found as the optimum elution condition. The calculated percent recovery of the imprinted polymer showed no considerable decrease

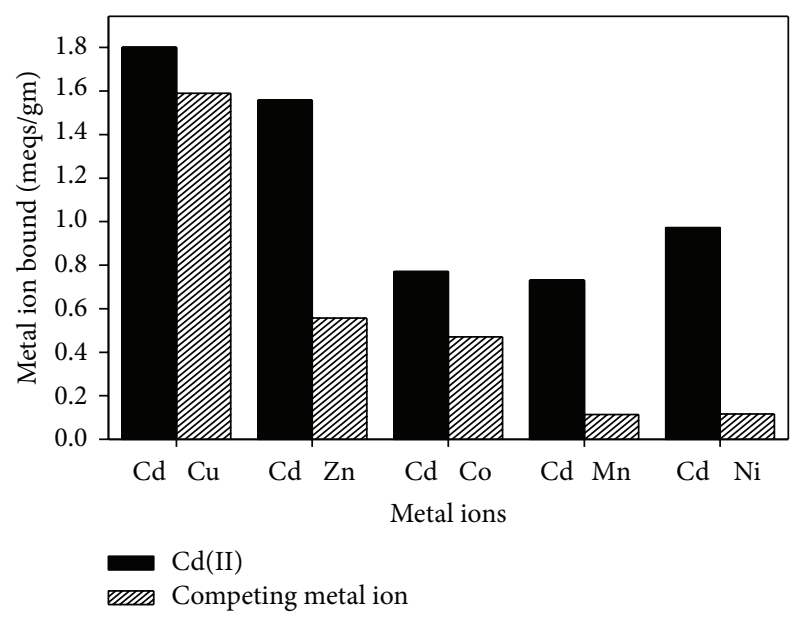

FIGURE 12: Summary of the selectivity study of Cd(II) ion imprinted interpenetrating polymer networks.

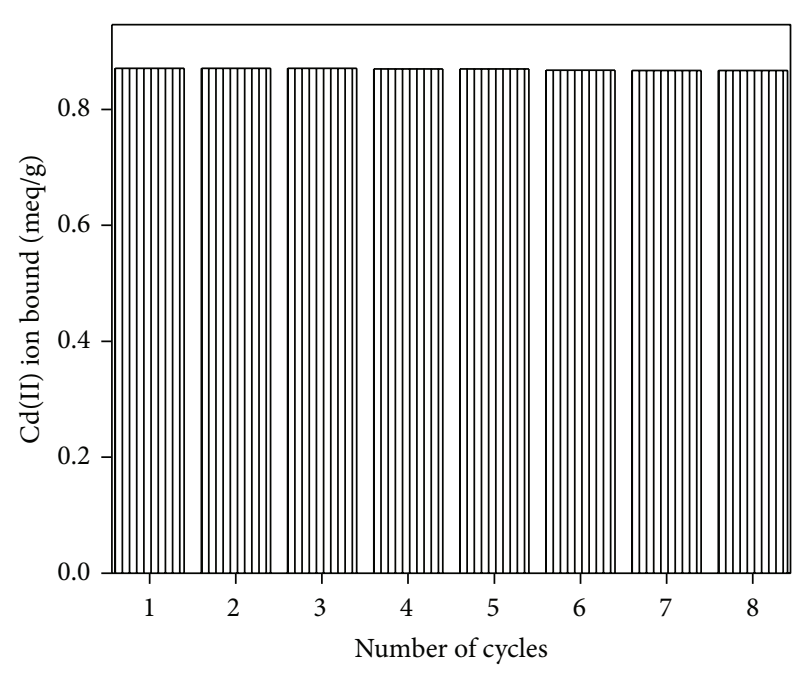

FIGURE 13: Reusability studies of Cd(II) ion binding by IIP.

after 8 cycles of repeated experiments. The percentage recovery of the recycled IIP could still be maintained at $98 \%$ at the 8 th cycle. It can be concluded that the Cd(II) ion imprinted polymer can be used for many times without significant decrease in its sorption capacity.

3.16. Analytical Precision and Detection Limits. Under the selected conditions, eight portions of standard solutions were enriched and analyzed simultaneously following the general procedure. The relative standard deviations (R. S. D.) of the method was lower than $2.2 \%$, which indicated that the method had good precision for the analysis of trace Cd(II) ion in solution samples. In accordance with the definition of IUPAC, the detectionlimit of the method wascalculated based on three times of the standard deviation of 11 runs of the blank solution. The detection limit $(3 \sigma)$ of the proposed method was $0.34 \mathrm{ng} \cdot \mathrm{mL}^{-1}$. 
TABLE 3: Comparative study of the Cd(II)IIP.

\begin{tabular}{|c|c|c|c|}
\hline Monomer & Polymerization technique & Adsorption capacity (meq/g) & Reference \\
\hline Epoxy resin triethylene tetra amine & Copolymerization & 0.0934 & 7 \\
\hline $\begin{array}{l}\text { 2,2-\{ethane-1,2-diylbis[nitrilo(E)metylylidene }]\} \text { diphenolate } \\
\text { 4-vinylpyridine }\end{array}$ & Suspension polymerization & 0.0042 & 18 \\
\hline Alginic acid acrylamide netwok & Free-radical polymerization & 0.217 & This study \\
\hline
\end{tabular}

TABLE 4: Analysis of environmental water samples.

\begin{tabular}{lcc}
\hline Cd(II) ion & Lake water $(\mathrm{mg} / \mathrm{L})$ & Canal water \\
\hline Found & 0.047 & 0.035 \\
Removed & 0.043 & 0.034 \\
Recovered (\%) & 98 & 99 \\
\hline
\end{tabular}

3.17. Comparison with Other Studied Polymers. In the literature, various ion imprinted polymers have been studied with a wide range of sorption capacities for $\mathrm{Cd}(\mathrm{II})$ ions. The results of this study are compared with them in Table 3 . The sorption capacities of the present polymers are varied in the range of $0.2-0.3 \mathrm{meqg}^{-1}$ from aqueous solutions. Therefore, the newly developed biosorbent exhibited better capacity values in comparison to most of the other alginic acid based and natural polymer sorbents.

3.18. Application of the Method. The synthesized IPN was applied to the analysis of $\mathrm{Cd}$ (II) ion in environmental water samples collected from Vembanad lake and nearby canals. The samples were treated by the same procedure. The samples were introduced into the cadmium ion imprinted polymer by column method and analysed by AAS. The results obtained indicate the suitability of the present $\mathrm{Cd}(\mathrm{II})$ ion imprinted IPN for the removal of hazardous Cd(II) ion from environmental water samples. The results were listed in Table 4 .

\section{Conclusions}

The present paper demonstrates the preparation of $\mathrm{Cd}(\mathrm{II})$ ion imprinted and nonimprinted interpenetrating polymer networks using functional polymer alginic acid and NNMBAcrosslinked polyacrylamide. The synthesised imprinted and nonimprinted interpenetrating polymer networks were characterized by FT-IR, UV-vis, SEM-EDAX, XRD, and TGA. The $\mathrm{Cd}(\mathrm{II})$ ion sorption was relatively fast. The maximum sorption capacity for $\mathrm{Cd}(\mathrm{II})$ ions was 0.8861 meqmole $^{-1}$ of imprinted polymer. The fast sorption equilibrium is most probably due to high complexation and geometric affinity between Cd(II) ions and the cavities in the network structure. The sorption values increased with increasing concentration of Cd(II) ions. Langmuir model was found to be applicable in interpreting $\mathrm{Cd}$ (II) ion sorption on the $\mathrm{Cd}$ (II) ion imprinted polymer and the adsorption kinetics was described by the pseudosecond-order kinetic model. Maximum swelling was obtained for $\mathrm{Cd}(\mathrm{II})$ ion imprinted and nonimprinted polymers rather than imprinted complexes. The sorption values increased with increase in $\mathrm{pH}$ and a saturation value was obtained at $\mathrm{pH}$ 6.9. Thermodynamic parameters were calculated using the van't Hoff equation and the sorption of $\mathrm{Cd}$ (II) ion on imprinted polymer networks was spontaneous and endothermic in nature and entropy of sorption increases during the reaction. Cd(II) ion imprinted interpenetrating polymer networks exhibited much high selectivity. The analytical results obtained in these investigations suggested that the sorbent may be used as an inexpensive and effective polymer for the removal of cadmium ion from aqueous solution and was successfully applied for the separation of $\mathrm{Cd}(\mathrm{II})$ ion from environmental water samples.

\section{Conflict of Interests}

The authors declare that there is no conflict of interests regarding the publication of this paper.

\section{Acknowledgment}

The author Girija Parameswaran thanks UGC Bangalore for providing a Teacher Fellowship.

\section{References}

[1] L. D. Mafu, T. A. M. Msagati, and B. B. Mamba, "Ionimprinted polymers for environmental monitoring of inorganic pollutants: synthesis, characterization, and applications," Environmental Science and Pollution Research, vol. 20, no. 2, pp. 790802, 2013.

[2] F. Aboufazeli, H. Zhad, O. Sadeghi, M. Karimi, and E. Najafi, "Novel Cd(II) ion imprinted polymer coated on multiwall carbon nanotubes as a highly selective sorbent for cadmium determination in food samples," Journal of AOAC International, vol. 97, no. 1, pp. 173-178, 2014.

[3] J. H. Kim, S. B. Lee, S. J. Kim, and Y. M. Lee, "Rapid temperature $/ \mathrm{pH}$ response of porous alginate-g-poly $(\mathrm{N}$ isopropylacrylamide) hydrogels," Polymer, vol. 43, no. 26, pp. 7549-7558, 2002.

[4] H. G. Seiler, A. Sigel, and H. Sigel, Handbook on Toxicity of Inorganic Compounds, Marcel Dekker, New York, NY, USA, 1998.

[5] Y. Zhai, Y. Liu, X. Chang, S. Chen, and X. Huang, "Selective solid-phase extraction of trace cadmium(II) with an ionic imprinted polymer prepared from a dual-ligand monomer," Analytica Chimica Acta, vol. 593, no. 1, pp. 123-128, 2007.

[6] K. Lu and X. P. Yan, "An imprinted organic-inorganic hybrid sorbent for selective separation of cadmium from aqueous solution," Analytical Chemistry, vol. 76, no. 2, pp. 453-457, 2004.

[7] J. Pan, S. Wang, and R. Zhang, "Ion-imprinted interpenetrating polymer networks for preconcentration and determination 
of Cd(II) by flame atomic absorption spectrometry," Chemia Analityczna, vol. 51, no. 5, pp. 701-713, 2006.

[8] S. E. Manahan, Environmental Chemistry, Lewis Publishers, Boca Raton, Fla, USA, 6th edition, 1994.

[9] J. S. Watson, Separation Methods for Waste and Environmental Applications, Marcel Dekker, New York, NY, USA, 1999.

[10] A. Martinsen, G. Skjak-Braek, and O. Smidsrod, "Alginate as immobilization material: I. Correlation between chemical and physical properties of alginate gel beads," Biotechnology and Bioengineering, vol. 33, no. 1, pp. 79-89, 1989.

[11] D. Solpan and M. Torun, "Investigation of complex formation between (sodium alginate/acrylamide) semi-interpenetrating polymer networks and lead, cadmium, nickel ions," Colloids and Surfaces A: Physicochemical and Engineering Aspects, vol. 268, no. 1-3, pp. 12-18, 2005.

[12] T. Alizadeh, "An imprinted polymer for removal of $\mathrm{Cd}^{2+}$ from water samples: optimization of adsorption and recovery steps by experimental design," Chinese Journal of Polymer Science, vol. 29, no. 6, pp. 658-669, 2011.

[13] S. Ozkara, M. Andac, and V. Karakoc, "Ion-imprinted PHEMA based monolith for the removal of $\mathrm{Fe}^{3+}$ ions from aqueous solutions," Journal of Applied Polymer Science, vol. 120, no. 3, pp. 1829-1836, 2011.

[14] P. Fan and B. Wang, "Regulatory effects of $\mathrm{Zn}$ (II) on the recognition properties of metal coordination imprinted polymers," Journal of Applied Polymer Science, vol. 116, no. 1, pp. 258-266, 2010.

[15] N. Burham, A. Mamdouh, and M. F. EL-Sahat, "Separation and determination of $\mathrm{Cd}^{2+}, \mathrm{Pb}^{2+}$ and $\mathrm{Cu}^{2+}$ from water samples using chemically modified groundnut shells," International Journal of Advanced Research, vol. 2, no. 1, pp. 755-765, 2014.

[16] B. George, V. N. R. Pillai, and B. Mathew, "Effect of the nature of the crosslinking agent on the metal-ion complexation characteristics of $4 \mathrm{~mol} \%$ DVB- and NNMBA-crosslinked polyacrylamide-supported glycines," Journal of Applied Polymer Science, vol. 74, no. 14, pp. 3432-3444, 1999.

[17] N. Sebastian, B. George, and B. Mathew, "Metal complexes of poly(acrylic acid): synthesis, characterization and thermogravimetric studies," Polymer Degradation and Stability, vol. 60, no. 2-3, pp. 371-375, 1998. 

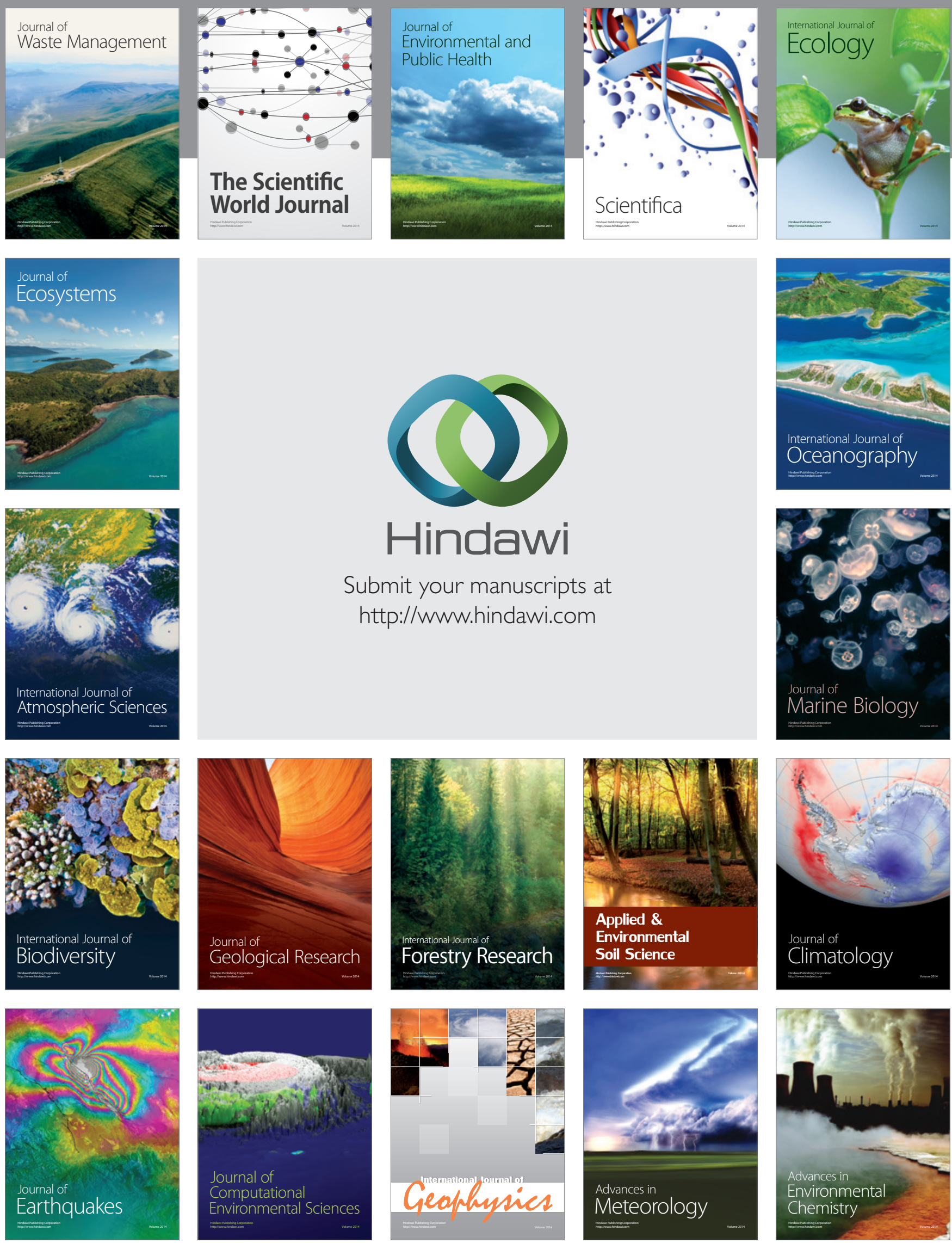